\title{
Absolute Distance Metrology Using Diode Pumped Nd:YVO$/$ KTP Green Laser
}

Mohamed Abdel Wahhab, ${ }^{1}$ Niveen Farid, ${ }^{1 *}$ Mohamed El-Okr ${ }^{2}$ and Mohamed El-Bahrawi ${ }^{1}$

${ }^{1}$ Length and Precision Engineering Division, National Institute for Standards,

Tersa St., Haram, Giza Code 12211, P.O. 136, Giza, Egypt

${ }^{2}$ Physics Department, Faculty of Science, Al-Azhar University,

Nasr City, P.O. 11651, Cairo, Egypt

*Corresponding author: niveen_farid@hotmail.com

Published online: 25 November 2019

To cite this article: Abdel Wahhab, M. et al. (2019). Absolute distance metrology using diode pumped Nd:YVO$/$ KTP green laser. J. Phys. Sci., 30(3), 1-10, https://doi. org/10.21315/jps2019.30.3.1

To link to this article: https://doi.org/10.21315/jps2019.30.3.1

\begin{abstract}
The paper exploits absolute distance measurement with traceability to the international system (SI) of units of length. In the present work, the diode pumped $\mathrm{Nd}: \mathrm{YVO}_{4} /$ KTP polarised green laser is used to measure a distance $\Delta l$ between two positions without the need for a reference standard. To comprise a synthetic wavelength $\lambda_{s}$ suitable to the range of the distance, the output wavelength generated by the frequency doubling is shifted by $\Delta \lambda$ by controlling the injection current, and a set of shifted wavelengths are produced to form $\lambda_{s}$. The output signal is sent to the interferometer by an optical fibre after being enhanced with a speckle removal unit. The measurement starts by identifying the zero position where no shift in the interference fringes is observed during sweeping the wavelengths, while at the distance $\Delta l$ a deviation in the interference pattern is observed. This deviation is analysed by Fourier transformation to extract the phase change $\Delta \phi$ and determine the distance value. To prove the reliability of absolute distance measuring interferometer, some measurements are made for various ranges with evaluation of both repeatability and uncertainty. The discussed technique can measure $100 \mathrm{~mm}$ with uncertainty $\pm 16 \mu \mathrm{m}$.
\end{abstract}

Keywords: Optical crystals, frequency doubling, tuneable wavelength, absolute measurement, absolute distance metrology 


\section{INTRODUCTION}

The absolute optical measurement does not rely on the external standard as it is based on quantum correlation of paired photons produced in a nonlinear medium. For example, in frequency doubling, sum, or difference, new correlated photons are generated from the input frequencies passing through the optical nonlinear crystals. Such correlated photons have been used widely by the meteorologists in the calibration of detector irradiance, optical frequencies and in spectroscopic applications without the need for calibrated standards. ${ }^{1-5}$

On the other hand, optical measurement of length based on defining the number of the interference fringes counted from a start position to an end position is a relative method, since it compares between two positions and determines the path between them. In the case of absolute measurement, the path between any two points within the coherence length of the superimposed waves can be measured without a reference position. Since accurate optical measurement depends on removing the ambiguity in the phase usually by using a synthetic wavelength that must be suitable to the path range, the scientists developed different methods for this purpose using different laser sources, dual frequency laser, or tuned diode laser. ${ }^{6-9}$ In addition, phase unwrapping technique is widely used in optical metrology for removing phase ambiguity. ${ }^{10}$

In this paper, Michelson interferometer is developed to be an absolute distance measuring interferometer using the tuneable $532 \mathrm{~nm}$ green laser generated by frequency doubling.

\section{ND:YVO 4 /KTP TUNABLE GREEN WAVELENGTH}

The laser active material, vanadate doped with neodymium atoms $\mathrm{Nd}: \mathrm{YVO}_{4}$ of $3 \%$ doped with dimension $3 \times 3 \times 1 \mathrm{~mm}$ is pumped by the $808 \mathrm{~nm}$ laser to generate the $1064 \mathrm{~nm}$ near infrared laser. The efficiency of the $\mathrm{Nd}: \mathrm{YVO}_{4}$ is due to the large absorption around the $808 \mathrm{~nm}$ peak pump wavelength that is suitable for the most available standard high power diode lasers. This means that a small size crystal is suitable for a compact laser system. Moreover, its structure as uniaxial crystal leads to a linearly polarised emission that avoids the undesirable birefringence effect on the frequency conversion. 
The input laser is $95 \%$ absorbed by the crystal and the output $1064 \mathrm{~nm}$ can be slowly shifted in the forward and the backward directions by $0.2 \mathrm{~nm}$ by controlling the injection current following the relationship:

$$
\lambda_{i, t}=\lambda_{i_{0}, t_{0}}+a \Delta t+b \Delta i
$$

where $i$ and $t$ represent the injection current and temperature respectively, while $a$ and $b$ are material constants.

The $2 \times 2 \times 5 \mathrm{~mm}$ frequency doubling crystal, potassium titanyl phosphate (KTP), generates the $532 \mathrm{~nm}$ laser in the resonant cavity. This crystal is widely used in the nonlinear optical systems for its high optical damage threshold, wide acceptance angle and high nonlinear coefficient. ${ }^{11}$ The high pumping efficiency is achieved by using a gradient index lens of focal length $3 \mathrm{~mm}$ to focus the $808 \mathrm{~nm}$ laser beam into $0.1 \mu \mathrm{m}$ spot and locate its waist inside the $\mathrm{Nd}: \mathrm{YVO}_{4}$ crystal. The back mirror of the resonant cavity bends when absorbing heat, and the front mirror is highly reflective to both $808 \mathrm{~nm}$ and $1064 \mathrm{~nm}$ lasers, while it is highly transparent to the $532 \mathrm{~nm}$ wavelength (Figure 1).

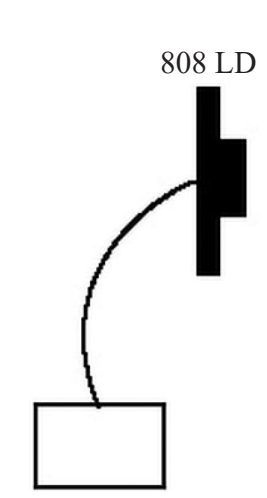

Current controller

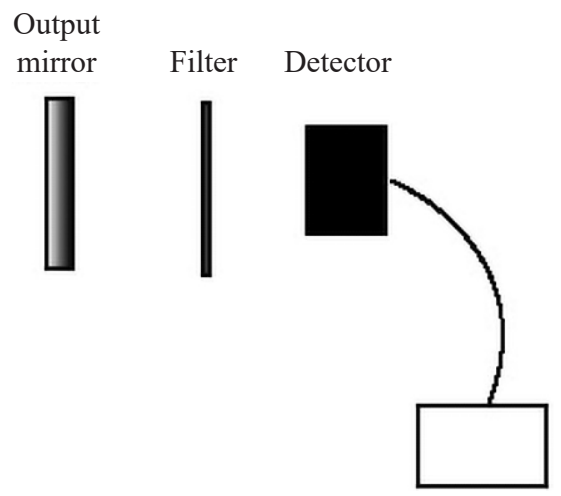

Power meter

Figure 1: Nonlinear Nd:YVO $/$ KTP system.

The tuning range of the wavelengths generated by the nonlinear effect and based on the injection current is found to be $1 \mathrm{~nm}$ as shown in Figure 2. This tuning range is determined experimentally with uncertainty $\pm 0.05 \mathrm{~nm}$ for six repeated measurements, and a synthetic wavelength can be comprised of a set of tuned wavelengths within this range that is quite effective to measure the required distance. 


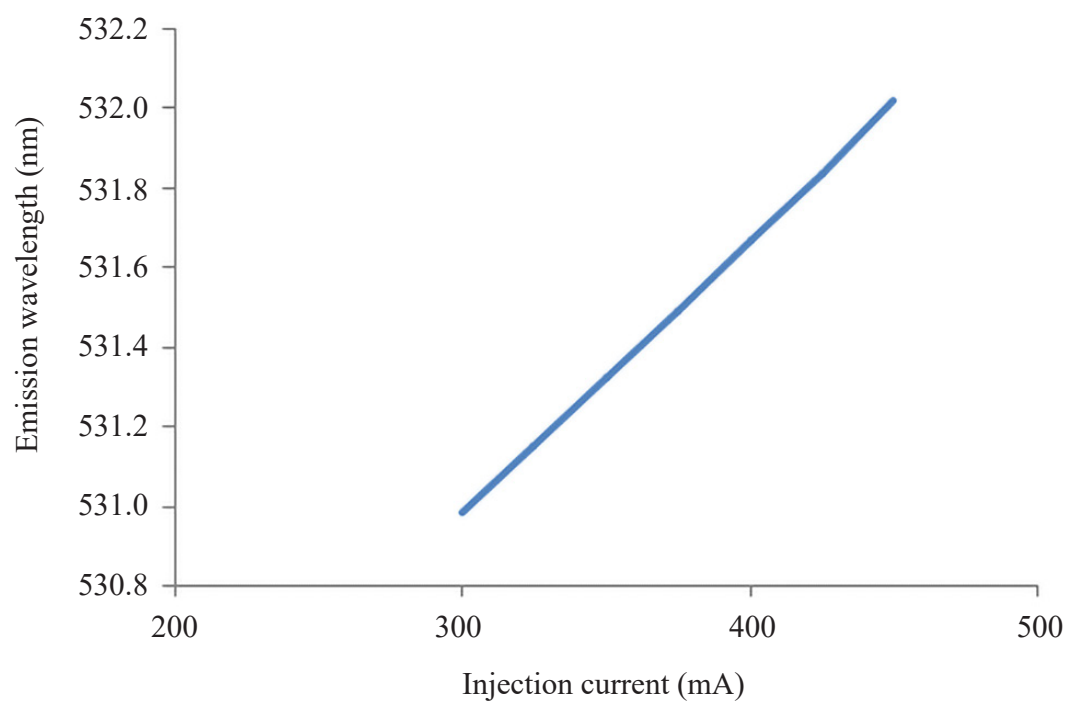

Figure 2: The tuning range of the green laser wavelength under the effect of the injection current in the diode laser.

\section{ADVANTAGES OF THE GREEN WAVELENGTH RADIATION}

The continuous green light is the most effective source in the dark areas as it is brighter than the red one and can transfer for long range in the dark when given a high output power. Moreover, its low sensitivity to the temperature variations and contribution to high beam quality and stability make it capable to measure long distances with low response to environmental effects. On the other hand, the charged coupled device (CCD) sensitivity is the best in the visible range as the quantum efficiency is high (90\%). Also, in near infrared (NIR) range, the sensitivity of the CCD matrix is relatively good, but beyond the $1100 \mathrm{~nm}$, no response will be found, and an (IR) detector has to be used.

Although red and blue laser diodes are commercially available, the continuous wave $(\mathrm{CW})$ green laser is not. Therefore, an alternative to the green diode laser is the $532 \mathrm{~nm} \mathrm{CW}$ green laser that is produced by the second harmonic generation, and it is well polarised, stabilised and has sufficient output power.

\section{ABSOLUTE DISTANCE INTERFEROMETER}

In Michelson interferometer, the light beam is divided by a beam splitter into two parts which are directed into two mirrors. In the reference case, both mirrors are placed at equal distance from the beam splitter. When beams are reflected back 
towards the beam splitter, they interfere, and interference patterns are produced. Once one of the two mirrors is displaced to another position by a distance $L$, the interference fringes are deviated from the reference case due to the change in the phase $(\Delta \phi)$ induced from this displacement.

The distance measurement using the conventional comparable method concerns the difference between the interference patterns produced at the zero and the end positions (Figure 3). In this case, the wavelength is a fixed value and the distance is calculated by counting the number of the interference fringes (integer + fraction) corresponding to the distance made by the mirror according to Equation 2:

$$
L+\Delta L=(n+\Delta n) \frac{\lambda}{2 \mu}
$$

where $n$ is the integer number of the fringes corresponding to the nominal value of the distance $L, \Delta n$ is the fringe fraction corresponding to the fraction in distance $\Delta l, \lambda$ is the wavelength used in the measurement, and $\mu$ is the refractive index of the surrounding air where the measurements are carried out. ${ }^{12,13}$

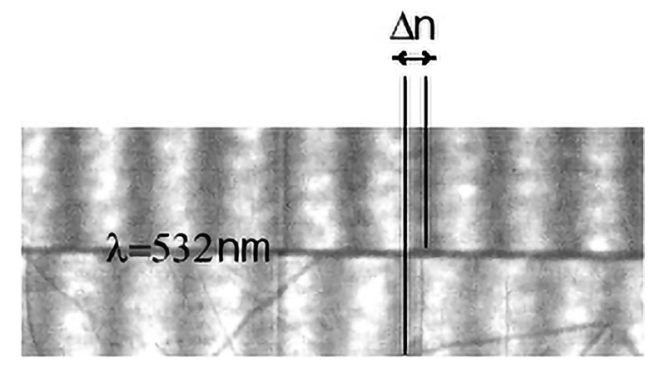

Figure 3: Interference patterns formed by the interferometer in the reference and end positions using $\lambda=532 \mathrm{~nm}$ by the comparable method.

The absolute measurement is mainly developed for industrial applications concerning calibration without need to a reference standard. In the absolute measurement, the mirror is kept fixed and the wavelength is shifted by $\Delta \lambda$ to different values $\lambda_{1}, \lambda_{2}, \ldots$, etc. A synthetic wavelength $\lambda_{\mathrm{s}}$ is comprised from $\lambda_{1}, \lambda_{2}$ such that it can suit the distance of interest $\Delta l$ :

$$
\lambda_{s}=\frac{\lambda_{1} \lambda_{2}}{\lambda_{2}-\lambda_{1}} \approx \frac{\left(\lambda_{1}\right)^{2}}{\delta \lambda}
$$

and $\Delta l$ is expressed by:

$$
\Delta l=N \lambda_{s}+\frac{\Delta \phi}{2 \pi} \lambda_{s}
$$


To identify the zero position $(\Delta l=0), \lambda_{1}$ produces the fundamental interference fringes, and $\lambda_{2}$ is then selected such that no deviation in the interference fringes is observed (i.e., $\Delta \phi=0)$. When the mirror is moved to a different position $(\Delta l \neq 0)$, a phase change is observed between the interference fringes produced by $\lambda_{1}$ and $\lambda_{2}$ (i.e., $\Delta \phi \neq 0$ ). By measuring $\Delta \phi$, the distance $\Delta l$ can be determined with accuracy depending on the stability of the wavelength, the resolution of the imaging sensor, and the resolution of the fringe analysis.

\section{MEASUREMENT}

The set of wavelengths within the tuning range of the $\mathrm{Nd}: \mathrm{YVO}_{4} / \mathrm{KTP}$ laser are calibrated with accuracy $\approx 10^{-9} \mathrm{~m}$. Increasing the injection current induces small deviation $\Delta \lambda$ to the previous value and a new wavelength $(\lambda+\Delta \lambda)$ is obtained. Assuming that the nominal distance can be measured by some mechanical means with accuracy $\pm \varepsilon$ (approximately $\pm 0.25 \mathrm{~mm}$ ), then the synthetic wavelength must be $\geq 2 \varepsilon$ (i.e., $\lambda_{\mathrm{s}} \geq 0.5 \mathrm{~mm}$ ). The first wavelength $\lambda_{1}=531.32 \mathrm{~nm}$ produces the fundamental interference pattern and the first interferogram is recorded. The second wavelength $\lambda_{2}$ is calculated from Equation 3 with deviation $\Delta \lambda=0.47 \mathrm{~nm}$, and the required value is achieved by adjusting the current controller such that $\lambda_{2}=531.79 \mathrm{~nm}$, then the second interference pattern is obtained.

The signal is filtered from the speckle noise by a fibre shaker unit which vibrates the output speckles by rapid shifting of the electromagnetic modes in the fibre. This high vibration mixes the modes and then the modes cannot be discriminated by the detector or the human eye and consequently the image appears smooth. ${ }^{14}$ The signal is sent to the interferometer input to measure the distances $(0.5 \mathrm{~mm}$, $10 \mathrm{~mm}, 50 \mathrm{~mm}, 80 \mathrm{~mm}$ and $100 \mathrm{~mm}$ ) made by the mirror.

Both $\lambda_{1}$ and $\lambda_{2}$ have to identify the zero position where the interference fringes formed by both wavelengths are in the same position and no deviation is observed, as shown in Figure 4(a). When shifting the mirror by $\Delta l$, a phase change $\Delta \phi$ comes to appear between the interference fringes formed by $\lambda_{1}$ and $\lambda_{2}$, seen in Figure 4(b).

The phase change $\Delta \phi$ between the shifted fringes formed by $\lambda_{1}, \lambda_{2}$ at distance $\Delta l$ is extracted by Fourier transformation to determine and the corresponding fringe fraction $\Delta n$. The accuracy of measuring the nominal length is necessary to determine the integer number of the interference fringes $N$. For a distance $100 \mathrm{~mm}$, the nominal value of $\Delta l$ is measured by a mechanical mean with accuracy $\pm 0.25 \mathrm{~mm}$ (i.e., $99.75<\Delta l<100.25$ ), and the distance measured by the absolute distance interferometer is calculated as $\Delta l=99.9974 \mathrm{~mm}$. 
(a)

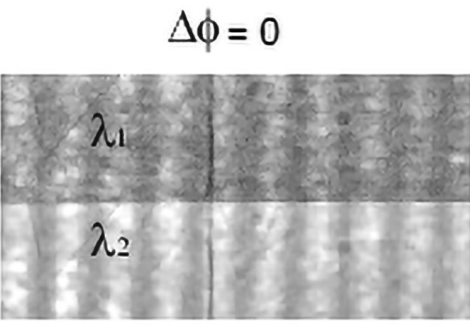

Fringes at the start point by $\lambda_{1}, \lambda_{2}$ (b)

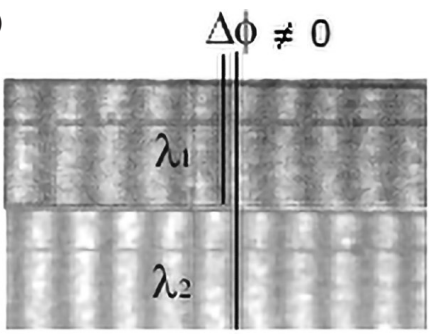

Fringes at the end point by $\lambda_{1}, \lambda_{2}$

Figure 4: Interference fringes formed by Michelson interferometer using $\lambda_{1}=531.32 \mathrm{~nm}$ and $\lambda_{2}=531.79$ at (a) zero position "start point," and (b) distance $\Delta l$ "end point."

\section{RESULTS AND DISCUSSION}

Based on the phase matching condition in the frequency doubling crystal KTP, the output wavelength is exactly half the input one. The wavelengths within the tuning range are calibrated separately with accuracy $\approx 10^{-9}$. Amongst the tuning range, two wavelengths $\left(\lambda_{1}=531.32 \mathrm{~nm}, \lambda_{2}=531.79 \mathrm{~nm}\right)$ are used to comprise a synthetic wavelength to measure the distance of interest. The $\mathrm{Nd}: \mathrm{YVO}_{4} / \mathrm{KTP}$ output laser is aligned to the interferometer input and the interference fringes are analysed. Figure 5 shows the results of various distances measured by the absolute distance interferometer, and Figure 6 shows the repeatability of the results.

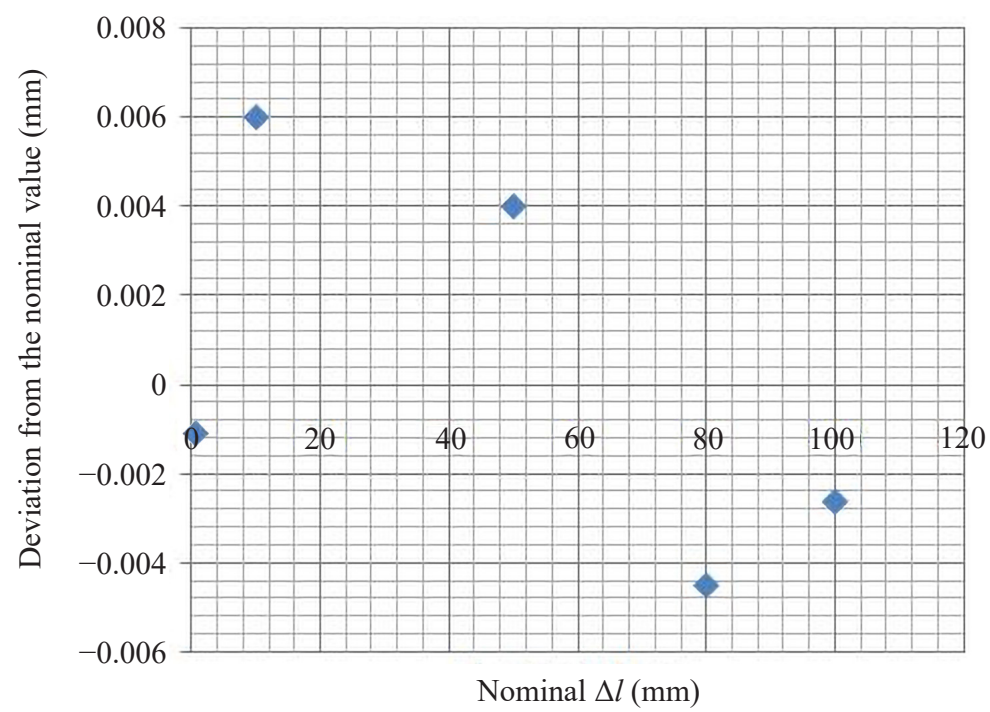

Figure 5: Results obtained by the absolute distance interferometer for the distance $\Delta l$ at $0.5 \mathrm{~mm}, 10 \mathrm{~mm}, 50 \mathrm{~mm}, 80 \mathrm{~mm}$ and $100 \mathrm{~mm}$. 


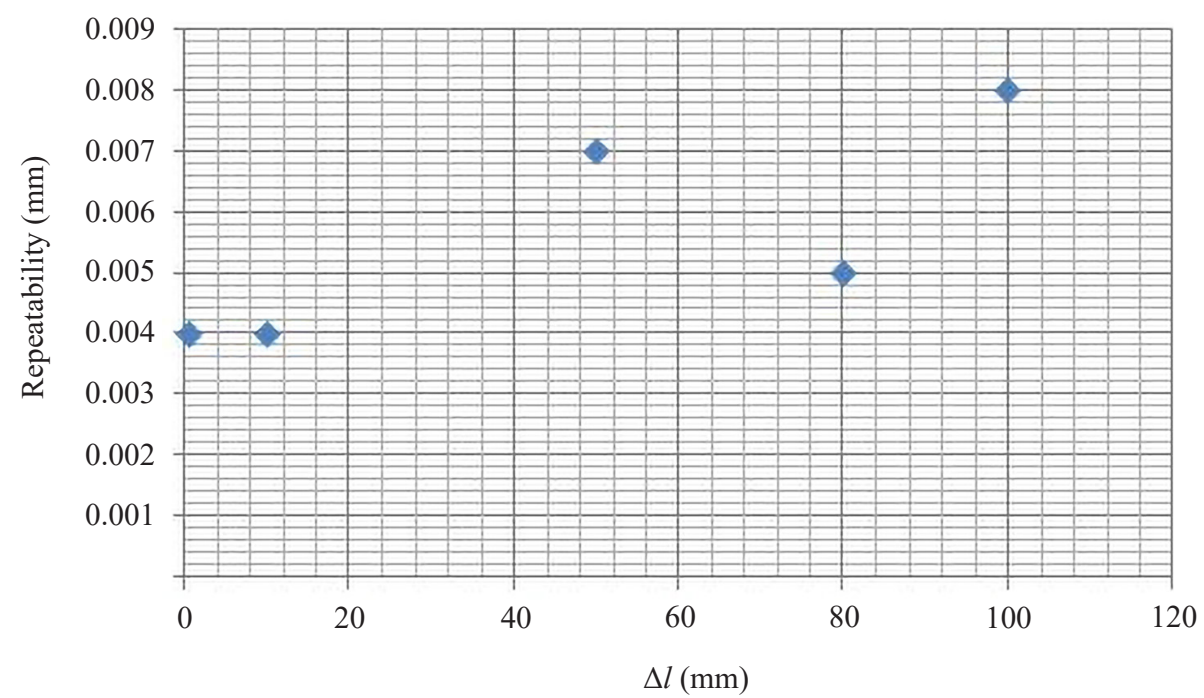

Figure 6: Repeatability of the set of measurements at $0.5 \mathrm{~mm}, 10 \mathrm{~mm}, 50 \mathrm{~mm}, 80 \mathrm{~mm}$ and $100 \mathrm{~mm}$.

The major contributors to the uncertainty in measurement are the stability of the tuned wavelength $\left(10^{-9} \mathrm{~m}\right)$ which contributes the uncertainty by $0.006 \Delta l \mu \mathrm{m}$, and the accuracy of $\Delta \phi(0.06$ radian $)$ which contributes the uncertainty by $0.002 \mu \mathrm{m}$. Thermal effects are also of a great concern as they alter the wavelength in use and contribute the uncertainty by $0.13 \Delta l \mu \mathrm{m}$. The repeatability in measurement is $8 \mu \mathrm{m}$ and the expanded uncertainty in measuring $(0.1 \mathrm{~m})$ distance is $\pm 16 \mu \mathrm{m}$. Table 1 summarises the uncertainty in measurement.

Table 1: Uncertainty in measuring the distance $\Delta l$ using the absolute distance interferometer.

\begin{tabular}{lcccc}
\hline $\begin{array}{l}\text { Source } \\
x_{i}\end{array}$ & $\begin{array}{c}\text { Limit } \\
\Delta x_{i}\end{array}$ & $\begin{array}{c}\text { Standard } \\
\text { uncertainty } \\
u\left(x_{i}\right)\end{array}$ & $\begin{array}{c}\text { Sensitivity } \\
\text { coefficient } \\
C_{i}\end{array}$ & $\begin{array}{c}\text { Uncertainty } \\
\text { contribution } \\
u\left(y_{i}\right) \mu \mathrm{m}\end{array}$ \\
\hline Wavelength stability & $6 \times 10^{-9} \mathrm{~m}$ & $3 \times 10^{-9} \mathrm{~m}$ & $\Delta l / \lambda$ & $0.006 \Delta l$ \\
$\Delta \phi$ & 0.006 radian & $0.002 \mu \mathrm{m}$ & 1 & 0.002 \\
Temperature & $0.2^{\circ} \mathrm{C}$ & $0.2^{\circ} \mathrm{C}$ & $9.6 \times 10^{-7} \Delta l$ & $0.13 \Delta l$ \\
Tuning effect & - & $0.05 \mathrm{~nm}$ & 1 & 0.00005 \\
Repeatability & - & $8 \mu \mathrm{m}$ & 1 & 8 \\
\hline
\end{tabular}

Notes: The combined uncertainty $u_{c}\left(y_{i}\right)=8 \mu \mathrm{m}$ at distance $0.1 \mathrm{~m} ;$ the expanded uncertainty $u_{95}(0.1 \mathrm{~m})=$ $\pm 16 \mu \mathrm{m}$. 


\section{CONCLUSION}

The concept of absolute measurement based on $\mathrm{Nd}: \mathrm{YVO}_{4} / \mathrm{KTP}$ tuneable green laser was exploited for the task of absolute measurement of distance. The tuning range of the green laser was calibrated with uncertainty $\approx 10^{-9} \mathrm{~m}$ and the wavelengths needed for the measurement could be selected amongst the tuning range by controlling the injection current. The synthetic wavelength $\lambda_{s}$ created by $\lambda_{1}$ and $\lambda_{2}$ had to be suitable to the distance of interest. The interference fringes produced by each wavelength were compared in order to extract the phase difference and determine the distance made by the interferometer mirror. The uncertainty in the absolute measurement of $L=100 \mathrm{~mm}$ was $\mathrm{U}_{95}=16 \mu \mathrm{m}$. The repeatability of the measurements reflects the performance of the developed absolute distance interferometer.

\section{ACKNOWLEDGEMENTS}

This work was supported by the National Institute for Standards, Egypt and the experimental measurements were done at the laboratory of end and line standards.

\section{REFERENCES}

1. Migdall, A. (1999). Correlated photon metrology without absolute standards. Phys. Tod., 52(1), 41-46, https://doi.org/10.1364/OE.23.016372.

2. Noorma, M. et al. (2003). Characterization of filter radiometers with a wavelengthtunable laser source. Metrol., 40(1), 220-223.

3. Brown, S., Eppeldauer, G. \& Lykke, K. (2006). Facility for spectral irradiance and radiance responsivity calibrations using uniform sources. Appl. Opt., 45(32), 8218-8237, https://doi.org/10.1364/AO.45.008218.

4. Anderson, V., Fox, N. \& Nettleton, D. (1992). Highly stable, monochromatic and tunable optical radiation source, and its application to high accuracy spectrophotometry. Appl. Opt., 31(4), 536-545, https://doi.org/10.1364/ AO.31.000536.

5. Hussein, H., Farid, N. \& Terra, O. (2015). Absolute gauge block calibration using ultra-precise optical frequency synthesizer locked to a femtosecond comb. Appl. Opt., 54(4), 622-626, https://doi.org/10.1364/AO.54.000622.

6. Bahrawi, M. \& Farid, N. (2012). Modified end standards interferometer and its performance in optical length metrology. Eng. e-Trans., 7(1), 1-5.

7. Farid, N. (2017). Automated contactless gauge block interferometer. J. Phys. Sci., 28(1), 87-98, https://doi.org/10.21315/jps2017.28.1.7.

8. Cabral, A., Abreu, M. \& Rebordão, J. (2009). Absolute distance metrology for long distance with dual frequency sweeping interferometry. Paper presented at the XIX IMEKO World Congress, Fundamental and Applied Metrology, Lisbon, 6-11 September. 
9. Abou-Zeid, A. \& Weise, P. (1998). Interferometer with a wavelength-tuned diode laser for surface profilometry. Meas. Sci. Tech., 9(7), 1105-1109.

10. Malacara, D., Servín, M. \& Malacara, Z. (2005). Phase unwrapping. In Malacara, D. (ed.), Interferogram analysis for optical testing, 2nd ed. New York: Taylor \& Francis, 499-531.

11. Jung, C. et al. (2009). A linearly-polarised Nd:YVO $/ \mathrm{KTP}$ microchip green laser. Opt. Exp., 17(22), 19611-19616, https://doi.org/10.1364/OE.17.019611.

12. Schodel, R. (2009). Length and size: Principles and applications. In Youshizawa, T. (ed.), Handbook of optical metrology. Tokyo: CRC Press, 744-750.

13. Bonch, G. \& Potulski, E. (1998). Measurement of the refractive index of air and comparison with modified Edlens formulae. Metrol., 35(2), 133-139.

14. Ha, W., Lee, S. \& Oh, K. (2008). Speckle reduction in near-field image of multimode fiber with a piezoelectric transducer. J. Opt. Soc. Kor., 12(3), 126-130, https://doi.org/10.3807/JOSK.2008.12.3.126. 